\title{
Laminin-332 mediates proliferation, apoptosis, invasion, migration and epithelial-to-mesenchymal transition in pancreatic ductal adenocarcinoma
}

\author{
CAIQUN HUANG ${ }^{1}$ and JUN CHEN ${ }^{2}$
}

Departments of ${ }^{1}$ Central Laboratory and ${ }^{2}$ Hepatobiliary and Pancreatic Surgery, Affiliated Jinhua Hospital, Zhejiang University School of Medicine, Jinhua, Zhejiang 321000, P.R. China

Received April 9, 2020; Accepted September 25, 2020

DOI: $10.3892 / \mathrm{mmr} .2020 .11649$

\begin{abstract}
The poor prognosis of patients with pancreatic ductal adenocarcinoma (PDAC) is primarily due to the invasive and metastatic behaviors of this disease. Laminin-332 (LM-332) is a key component of the basement membrane barrier, and is associated with tumor metastasis. The present study provides evidence towards the potential function of LM-332 in carcinoma, indicating the distinct roles of the three LM-332 subunits $(\alpha 3, \beta 3$ and $\gamma 2)$ in cell proliferation, migration, invasion, apoptosis and the epithelial-to-mesenchymal transition (EMT) in cancer. The roles of the $\alpha 3, \beta 3$ and $\gamma 2$ subunits in the malignant biological behavior of PDAC were investigated in the present study. It was revealed that the $\alpha 3, \beta 3$ and $\gamma 2$ subunits were upregulated in PDAC. Inhibition of all LM-332 subunits abrogated the tumorigenic outcomes, which included cell proliferation, apoptosis, invasion, migration and EMT in vitro. However, the three LM-332 subunits had different degrees of effects on biological behavior. It was observed that LAMA3 $(\alpha 3)$ had a stronger effect on cell proliferation, migration and invasion. In addition, LAMB3 ( $\beta 3$ ) knockdown significantly increased E-cadherin levels and decreased vimentin levels, indicating that LAMB3 was associated with EMT. Likewise, LAMC2 $(\gamma 2)$ mediated proliferation, apoptosis, invasion and migration. However, small interfering (si)-LAMC2 promoted the progression of EMT, which was the opposite effect to that of si-LAMB3. The LM-332 subunits $(\alpha 3, \beta 3$ and $\gamma 2)$ may be novel therapeutic targets of PDAC in the future.
\end{abstract}

Correspondence to: Professor Jun Chen, Department of Hepatobiliary and Pancreatic Surgery, Affiliated Jinhua Hospital, Zhejiang University School of Medicine, 351 Mingyue Road, Jinhua, Zhejiang 321000, P.R. China

E-mail:09chenjun@163.com

Key words: pancreatic ductal adenocarcinoma, laminin-332, laminin subunit $\alpha 3$, laminin subunit $\beta 3$, laminin subunit $\gamma 2$, epithelial-to-mesenchymal transition, biological behavior

\section{Introduction}

Pancreatic ductal adenocarcinoma (PDAC) is a common malignant tumor of the digestive tract, which is characterized by rapid progression, high mortality and high invasiveness $(1,2)$. It has been reported that $~ 50 \%$ of patients have been confirmed to possess metastasized cancer cells (3). Despite continuous improvements in diagnosis and treatment, this type of cancer is almost invariably fatal, and the 5-year survival rate of patients is $~ 8 \%(4,5)$. PDAC has a high degree of malignant biological characteristics, including invasion and metastasis, which leads to a poor prognosis. Therefore, revealing the molecular mechanisms underlying PDAC progression, and developing corresponding targeted therapies, is essential to improve the outcome of patients with PDAC.

Laminin is the main component of the extracellular matrix (ECM); it is located on the basement membrane and plays an important role in cell differentiation, adhesion and migration, as well as signal transduction (6,7). Laminin-332 (LM-332) is a primary member of the laminin family. It is composed of $\mathrm{LN} \alpha 3$, LN $\beta 3$ and $\mathrm{LN} \gamma 2$ chains, which are encoded by the LAMA3, $L A M B 3$ and $L A M C 2$ genes, respectively $(8,9)$. The three genes each express three strands, and the subsequent formation of a heterotrimer is a key step in the formation of the LM-332 complex. It has been demonstrated that LM-332 promotes various cell functions, including scattering, migration, polarization, adhesion, proliferation and apoptosis, through adhesion plaques and hemidesmosomes formed by interacting with integrin $\alpha 3 \beta 1$ and $\alpha 6 \beta 4(10,11)$. In addition, these integrins interact with adhesion molecules to affect signal transduction, which plays a key role in tumor invasion and metastasis. These characteristics of LM-332 suggest that it may play a key role in the cancerous transformation of normal cells.

The epithelial-to-mesenchymal transition (EMT) refers to the biological process in which epithelial cells are transformed into cells with a mesenchymal phenotype (12). EMT plays a vital role in tumor cell invasion and metastasis $(13,14)$. When tumor cells undergo EMT, the cell skeleton undergoes reorganization; the epithelial cell phenotype is lost; and the expression levels of proteins that enhance intercellular adhesion, such as E-cadherin, desmoplakin, zonula occludens-1 and claudin, are decreased. Simultaneously, molecular markers of interstitial cells, such as 
vimentin, fibronectin, matrix metalloproteinases and $\mathrm{N}$-cadherin, exhibit increased expression levels, resulting in decreased or lost adhesion between cells, loose connection between cells, loss of polarity, increased migratory ability, anti-apoptotic effects and degradation of the ECM, which are important biological processes for tumor invasion and metastasis to distant organs $(15,16)$. By detecting the expression levels of classic markers associated with EMT in malignant tumors, their roles in the prognosis of malignant tumors can be clarified, and key molecules can be selected to screen patient populations with a high risk of metastasis and provide effective evidence for predicting prognosis and metastasis, and provide personalized treatment options.

The present study investigated the roles of LM-332 subunits $(\alpha 3, \beta 3$ and $\gamma 2)$ in the malignant biological behavior of PDAC. It was revealed that LAMA3, LAMB3 and LAMC2 were upregulated in PDAC cells. It could decrease cell proliferation, invasion and migration by inhibiting the expression of $\alpha 3, \beta 3$ and $\gamma 2$ subunits. The present study identified a novel molecular mechanism of action of LM-332 in PDAC, which may provide a novel therapeutic strategy to block PDAC invasion and metastasis.

\section{Materials and methods}

Cell culture. The human pancreatic cancer cell lines PANC1 and $\mathrm{BxPC} 3$, and the normal human pancreatic ductal epithelial cell line HPDE6c7 (H6C7) were purchased from The Cell Bank of Type Culture Collection of the Chinese Academy of Sciences. All cell lines were cultured in Dulbecco's modified Eagle's medium (Gibco; Thermo Fisher Scientific, Inc.) containing $10 \%$ fetal bovine serum (FBS; Gibco; Thermo Fisher Scientific, Inc.) in an incubator with $5 \% \mathrm{CO}_{2}$ at $37^{\circ} \mathrm{C}$.

RNA interference. Small interfering (si)RNA duplexes against LAMA3 (5'-GCACAAACATTAAACAACA-3'), siRNA duplexes against LAMB3 (5'-GATGGAGGAAGATGTC AGA-3'), siRNA duplexes against LAMC2 (5'-GTCAAAGCCT GTCCTTTGA-3') and control duplex (si-NC; 5'-UUCUCCGAA CGUGUCACGU-3') were designed by Guangzhou RiboBio Co., Ltd. Cells not transfected with siRNA served as the control group. Cells were transfected with $50 \mathrm{nM}$ siRNA duplex using Lipofectamine ${ }^{\circledR}$ RNAiMAX (Invitrogen; Thermo Fisher Scientific, Inc.), according to the manufacturer's instructions. PANC1 cells ( $1 \times 10^{6}$ cells/well) were cultured in 6-well plates and were transfected with siRNA for $48 \mathrm{~h}$.

Reverse transcription-quantitative PCR (RT-qPCR). For the RT-qPCR analysis, total RNA was isolated from H6C7, PANC1 and BxPC3 cells using TRIzol ${ }^{\circledR}$ reagent (Invitrogen; Thermo Fisher Scientific, Inc.), according to the manufacturer's protocol. cDNA was synthesized from total RNA $(2 \mu \mathrm{g})$ using iScript ${ }^{\mathrm{TM}}$ cDNA Synthesis Kit (Bio-Rad Laboratories, Inc.). Amplification

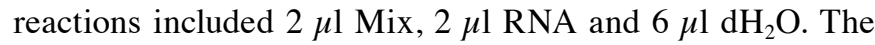
temperature protocol was as followed: $37^{\circ} \mathrm{C}$ for $15 \mathrm{~min}, 85^{\circ} \mathrm{C}$ for $5 \mathrm{sec}$ and $4^{\circ} \mathrm{C}$ for $10 \mathrm{~min}$. qPCR was performed with an ABI PRISM 7900 Sequence Detection System (Applied Biosystems; Thermo Fisher Scientific, Inc.) using the $\mathrm{iTaq}^{\mathrm{TM}}$ Universal SYBR-Green Supermix (Bio-Rad Laboratories, Inc.). Amplification reactions included $1 \mu \mathrm{l}$ cDNA template, $0.3 \mu \mathrm{l}$ each of the forward and reverse primers (10 $\mu \mathrm{M}), 0.2 \mu 150 \mathrm{X}$
ROX Reference Dye II (Takara Biotechnology Co., Ltd.), and $5 \mu 12 X$ SYBR Premix DimerEraser in a total volume of $10 \mu \mathrm{l}$. The following primer sequences were used: $\beta$-actin forward, 5'-ACTCTTCCAGCCTTCCTTCC-3' and reverse, 5'-CGTCAT ACTCCTGCTTGCTG-3'; LAMA3 forward, 5'-AAAGCGTAT GTGGATAAATGTGG-3' and reverse, 5'-CGGAAAGCAGGC GTAGAAA-3'; LAMB3 forward, 5'-GGCAGATGATTAGGG CAGCCGAGGAA-3' and reverse, 5'-CGGACCTGCTGGATT AGGAGCCGTGT-3'; LAMC2 forward, 5'-TTCTACAACGAT CCGCACGAC-3' and reverse, 5'-ACACCACCTCCTCCGTC TCC-3'. Amplification of the transcripts involved an initial denaturation at $95^{\circ} \mathrm{C}$ for $30 \mathrm{sec}$, followed by 40 cycles at $95^{\circ} \mathrm{C}$ for $5 \mathrm{sec}, 55^{\circ} \mathrm{C}$ for $30 \mathrm{sec}$, and $72^{\circ} \mathrm{C}$ for $34 \mathrm{sec}$. The cycle quantification $(\mathrm{Cq})$ comparison method was used for relative quantification. $\beta$-actin was used as the internal control for normalization. All qPCR analyses were performed in triplicate. Results were calculated using the $2^{-\Delta \Delta \mathrm{Cq}}$ method (17).

Western blot analysis. Proteins extracted from H6C7, PANC1 and $\mathrm{BxPC} 3$ cells using radioimmunoprecipitation assay lysis buffer (Beyotime Institute of Biotechnology) containing $1 \mathrm{mM}$ phosphatase and protease inhibitors. The protein concentration was quantified using a bicinchoninic protein assay kit (Beyotime Institute of Biotechnology), and then $20 \mu$ g protein were separated by sodium dodecyl sulfate polyacrylamide gel electrophoresis ( 8 to $12 \%$ gels) and transferred to polyvinylidene difluoride membranes (Merck KGaA). The membrane was blocked with $5 \%$ skimmed milk powder at room temperature for $2 \mathrm{~h}$. Then, the membrane was incubated with primary antibodies against LAMB3 (1:2,000; cat. no. LAMB3:26795-1-AP; ProteinTech Group, Inc.) LAMC2 (1:2,000; cat. no. LAMC2:19698-1-AP; ProteinTech Group, Inc.), E-cadherin (1:2,000; cat. no. E-cadherin:20874-1-AP; ProteinTech Group, Inc.) vimentin (1:2,000; cat. no. Vimentin:10366-1-AP; ProteinTech Group, Inc.) and LAMA3 (1:1,000; cat. no. ab151715; Abcam) overnight at $4^{\circ} \mathrm{C}$. Primary antibodies were detected with a HRP-conjugated goat anti-mouse $\operatorname{IgG}$ (1:5,000; cat. no. A0216) or goat anti-rabbit IgG (1:5,000; cat. no. A0218; both Beyotime Institute of Biotechnology) secondary antibodies for $2 \mathrm{~h}$ at room temperature. Proteins were visualized using an enhanced chemiluminescence kit (Merck KGaA) according to the manufacturer's instructions. Protein bands were normalized to $\alpha$-tubulin and protein expression was semi-quantified by ImageJ (version 1.8.0; National Institutes of Health).

Cell viability assay. A total of $3 \times 10^{3} \mathrm{PANC} 1$ cells were seeded in 96-well plates overnight. All cells were transfected with siRNA for 2 days. Cell Counting Kit-8 (CCK-8) solution (Beyotime Institute of Biotechnology) was added to each well and incubated for $2 \mathrm{~h}$ according to the manufacturer's instructions. The absorbance was measured using a BioTek ${ }^{\circledR}$ Synergy ${ }^{\mathrm{TM}}$ HT reader (BioTek Instruments, Inc.) at $450 \mathrm{~nm}$. All assays were repeated in triplicate.

Colony formation assay. An initial density of 500 cells/well were seeded in 6-well plates, and cultured for 2 weeks. The cells were fixed with $4 \%$ paraformaldehyde for $30 \mathrm{~min}$ at room temperature and stained with $0.1 \%$ crystal violet for $1 \mathrm{~h}$ at room temperature (Sigma Aldrich; Merck KGaA). Colonies containing >50 cells were counted using a light microscope at x10-20 magnification. 

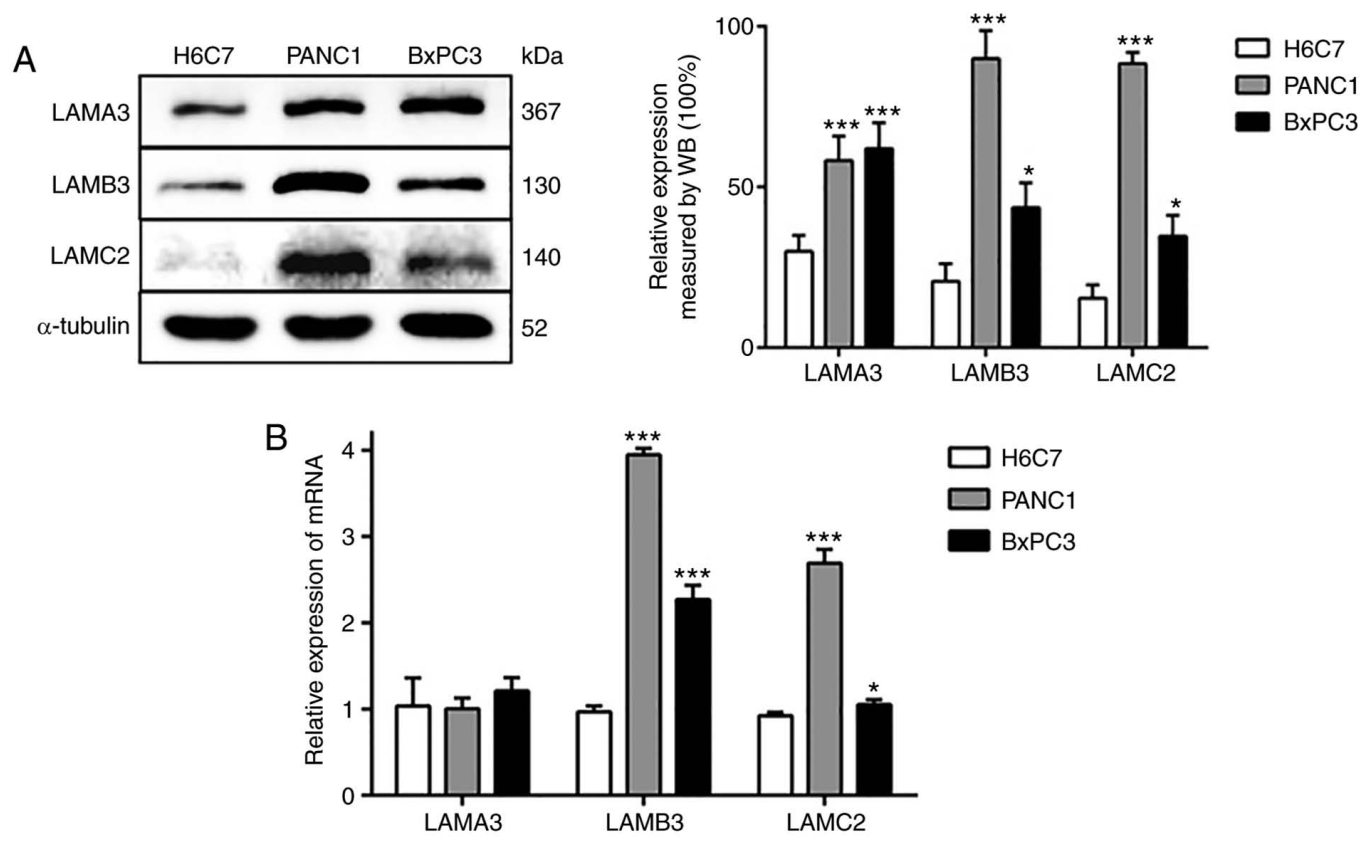

Figure 1. Expression of $\alpha 3, \beta 3$ and $\gamma 2$ chains of LM-332 in human pancreatic ductal epithelial and pancreatic cancer cell lines. (A) A normal pancreatic cell line and two pancreatic cancer cell lines (PANC-1 and BXPC-3) were prepared and examined via WB analysis. (B) The expression levels of LAMA3, LAMB3 and LAMC2 were examined via reverse transcription-quantitative PCR. Each experiment was performed in triplicate. ${ }^{*} \mathrm{P}<0.05,{ }^{* * * *} \mathrm{P}<0.01$ vs. $\mathrm{H} 6 \mathrm{C} 7$ group. LM-332, laminin-332; LAMA3, laminin subunit $\alpha 3$; LAMB3, laminin subunit $\beta 3$; LAMC2, laminin subunit $\gamma 2$; WB, western blotting.

Cell invasion and migration assays. The Transwell membrane (Corning, Inc.) was coated with Matrigel for $2 \mathrm{~h}$ at room temperature during the invasion measurement, and Matrigel was not used for the migration measurement. PANC1 cells $\left(5 \times 10^{4}\right)$ were seeded into the upper chambers in serum-free medium, and $600 \mu \mathrm{l}$ medium containing $20 \%$ FBS was added to the lower chambers. After incubation for $24 \mathrm{~h}$, the cells adhering to the upper surface of the membrane were removed. The invaded or migrated cells that had adhered to the lower surface were stained with $0.1 \%$ crystal violet for $1 \mathrm{~h}$ at room temperature and calculated by counting the number of cells in nine random fields of view using a fluorescence microscope at x10-20 magnification,.

Cell apoptosis analysis. After transfection of PANC1 cells for $48 \mathrm{~h}$, the cells were digested with trypsin and then washed twice with cold phosphate buffered saline to collect the cells. Subsequently, according to the manufacturer's instructions, using a FITC Annexin V Apoptosis Detection Kit (Beyotime Institute of Biotechnology), Annexin V FITC and propidium iodide were used to double stain the cells. The percentage of early + late apoptotic cells was analyzed by a BD FACSCanto ${ }^{\mathrm{TM}}$ II (BD Biosciences) flow cytometer using BD FACSDiva software (version 6.1.3; BD Biosciences).

Statistical analysis. All experiments were performed in triplicate. All values were presented as the mean \pm SD. GraphPad Prism 6.02 (GraphPad Software, Inc.) was used to determine the statistical significance of differences in the means of experimental groups. A paired Student's t-test was used to analyze the statistical significance between two groups of data. One-way ANOVA followed by a Student-Newman-Keuls post hoc test were used for multiple comparisons. $\mathrm{P}<0.05$ was considered to indicate a statistical significant difference.

\section{Results}

$\alpha 3, \beta 3$ and $\gamma 2$ chains of LM-332 are upregulated in PDAC cells. In order to determine the expression levels of the $\alpha 3, \beta 3$ and $\gamma 2$ chains of LM-332, the present study assessed LAMA3, LAMB3 and LAMC2 expression levels in two PDAC cell lines (PANC1 and BxPC3) and a human immortalized, non-tumorigenic pancreatic ductal epithelial cell line (H6C7). LAMB3 and LAMC2 expression levels were significantly increased in PANC1 and BxPC3 cells, both at the protein and mRNA levels (Fig. 1). In addition, the protein expression level of LAMA3 in the PDAC cell line was increased compared with the normal pancreatic cell line. However, there was no difference in the mRNA expression of LAMA3. These results indicated that the expression of LAMA3, LAMB3 and LAMC2 in the PDAC cell line is increased (particularly the level of LAMB3) compared with the normal pancreatic cell line. Subsequently, the present study selected PANC1 cells for the subsequent experiments, as there were higher differences in the expression levels of LAMB3 and LAMC2 when compared with the findings in BxPC3 cells.

Knockdown of LAMA3 inhibits proliferation, migration and invasion, promotes apoptosis, and regulates EMT in PANC1 cells. To further investigate the function of LAMA3, the present study used siRNAs designed specifically against LAMA3 to test the biological behavior of PDAC cells. The CCK- 8 and colony formation assays were used to detect cell proliferation. Transwell migration and invasion assays were used to detect cell migratory and invasive abilities, respectively. Flow cytometry was used to investigate whether LAMA3 promoted PDAC proliferation by regulating cell apoptosis. The RT-qPCR results confirmed that transfection with si-LAMA3 led to the downregulation of LAMA3 in PANC1 cells when compared with the findings in si-NC cells (Fig. 2A). The CCK-8 assay 

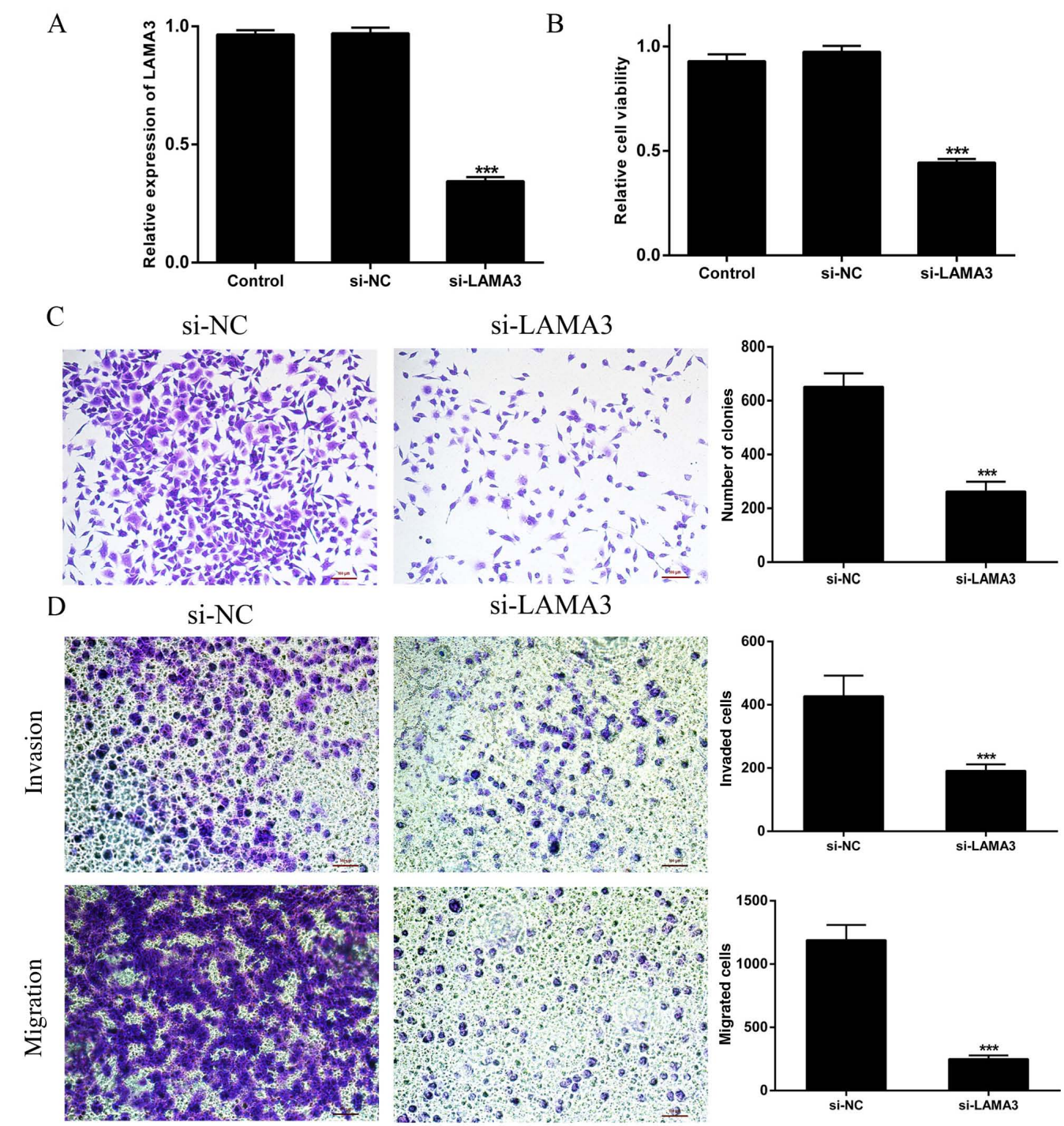

$\mathrm{E}$
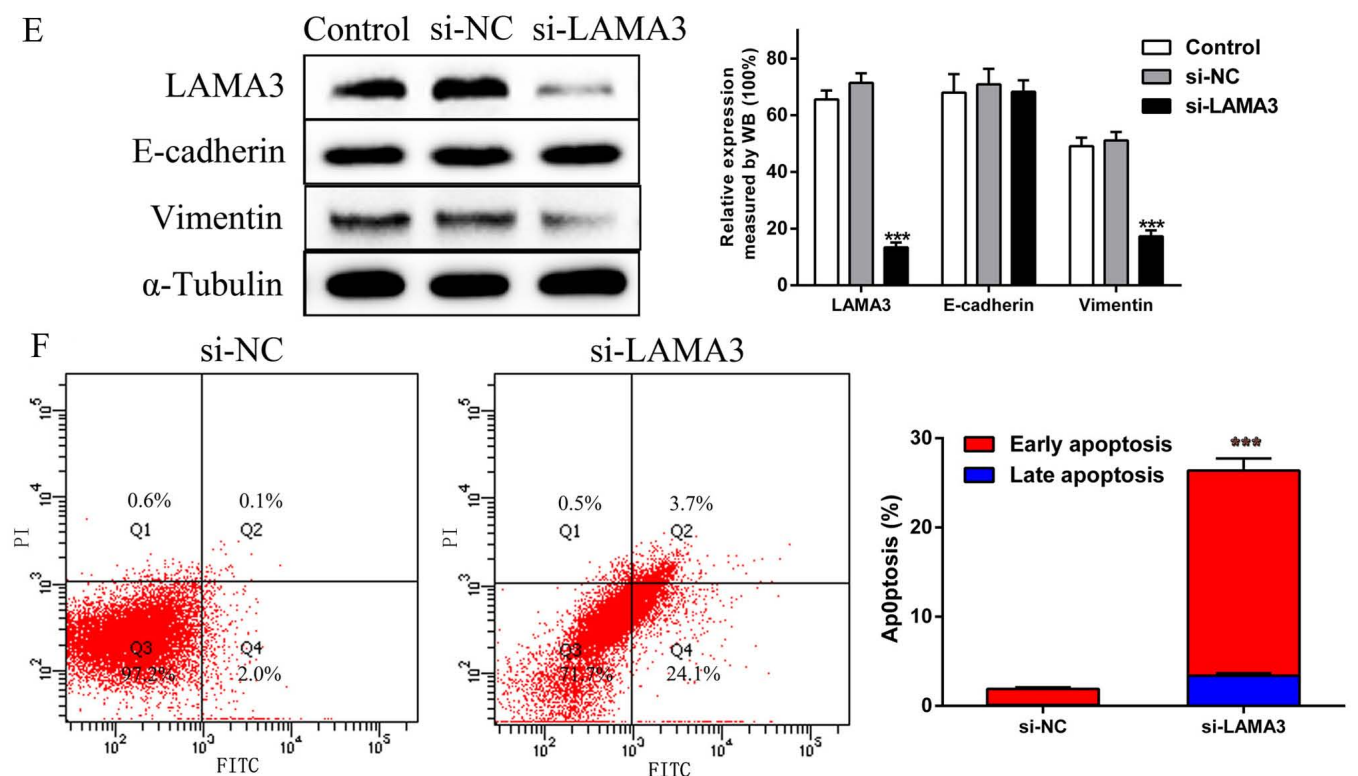

Figure 2. Knockdown of LAMA3 inhibits PDAC cell proliferation, migration, invasion and EMT, and promotes PDAC cell apoptosis. (A) LAMA3 mRNA expression was evaluated via reverse transcription-quantitative PCR in PANC-1 cells transfected with si-LAMA3. (B) Cell proliferation was analyzed by Cell Counting Kit-8 assays in the Control, si-NC and si-LAMA3 groups. (C) Colony formation assays were performed in the si-NC and si-LAMA3 groups. (D) Transwell invasion and migration assays. (E) EMT-associated proteins E-cadherin and vimentin were examined via WB analysis in the Control, si-NC and si-LAMA3 groups. (F) The percentage of apoptotic cells was demonstrated by flow cytometry in the si-NC and si-LAMA3 groups. Scale bar, $100 \mu \mathrm{m}$. ${ }^{* * * *} \mathrm{P}<0.01$ vs. si-NC group. PDAC, pancreatic ductal adenocarcinoma; EMT, epithelial-to-mesenchymal transition; siRNA, small interfering RNA; NC, negative control; LAMA3, laminin subunit $\alpha 3$; WB, western blotting. 
demonstrated that the viability of PANC1 cells transfected with si-LAMA3 was significantly impaired when compared with the findings in si-NC cells (Fig. 2B). Similarly, the results of the colony formation assay indicated that si-LAMA3 decreased the number of colonies (Fig. 2C). In addition, the migration and invasion assays demonstrated that the migratory and invasive abilities of PANC1 cells transfected with si-LAMA3 were significantly reduced (Fig. 2D). To be certain of the requirement of LAMA3 for EMT, western blotting was used to evaluate the EMT-associated protein vimentin and the epithelial marker E-cadherin. It was revealed that si-LAMA3 significantly reduced vimentin expression, but there was no difference in E-cadherin expression when compared with the findings in si-NC cells (Fig. 2E). Simultaneously, in LAMA3-knockdown cells, the number of apoptotic cells increased, particularly those in the early stages of apoptosis (Fig. 2F). Overall, these results indicated that LAMA3 knockdown could inhibit proliferation, migration and invasion; promote apoptosis; and regulate EMT in PDAC cells.

Knockdown of LAMB3 inhibits proliferation, migration and invasion, promotes apoptosis, and regulates EMT in PANC1 cells. To confirm the requirement of LAMB3 in the biological behavior of tumor cells, si-LAMB3 was transfected into PANC1 cells. Its expression was significantly decreased when compared with the findings in si-NC cells (Fig. 3A). Likewise, cell proliferation was significantly reduced by si-LAMB3 (Fig. 3B and C). Similarly, si-LAMB3 decreased migration and invasion in PANC1 cells (Fig. 3D). The protein expression levels of E-cadherin and vimentin were assessed via western blotting. It was noted that the expression of E-cadherin was significantly higher, and the expression of vimentin was significantly lower in cells transfected with si-LAMB3 (Fig. 3E). Simultaneously, in LAMB3-knockdown cells, the number of apoptotic cells increased, particularly those in the early stages of apoptosis (Fig. 3F). These results revealed that LAMB3 knockdown could inhibit proliferation, migration and invasion; promote apoptosis; and regulate EMT in PDAC cells.

Knockdown of LAMC2 inhibits proliferation, migration and invasion, promotes apoptosis, and regulates EMT in PANC1 cells. The RT-qPCR results confirmed that transfection with LAMC2 siRNA led to the downregulation of LAMC2 in PANC1 cells when compared with the findings in si-NC cells (Fig. 4A). The CCK-8 assay indicated that the viability of LAMC2-knockdown PANC1 cells was significantly impaired when compared with the findings in si-NC cells (Fig. 4B). Likewise, the colony formation results showed that si-LAMC2 decreased the colony numbers (Fig. 4C). Furthermore, the migration and invasion assays showed that the migration and invasion of PANC1 cells with si-LAMC2 was significantly reduced (Fig. 4D). In addition, si-LAMC2 significantly decreased E-cadherin expression and increased vimentin expression when compared with that in si-NC cells (Fig. 4E). Furthermore, among cells transfected with si-LAMC2, the number of apoptotic cells increased, particularly those in the early stages of apoptosis (Fig. 4F). These results indicated that LAMC2 knockdown could inhibit proliferation, migration and invasion, promote apoptosis, and regulate EMT in PDAC cells.

\section{Discussion}

Among patients diagnosed with advanced metastatic disease, pancreatic cancer is one of the most malignant tumors and deadly types of cancer, due to the difficulty in early detection and lack of diagnostic and prognostic biomarkers $(18,19)$. Due to its highly malignant biological characteristics, pancreatic cancer has a poor prognosis (20). Although various comprehensive treatment methods have been enhanced in recent years, the 5-year survival rate of patients remains very low (21). It is necessary to elucidate the invasion and metastasis mechanisms underlying pancreatic cancer in order to improve the current treatment situation.

A number of previous studies have demonstrated that adhesion and extracellular matrix proteins promote the development of various solid tumors, including pancreatic cancer (22-24). Metastasis is a deadly feature of cancer, requiring the invasion of cells through the basement membrane, which usually acts as a barrier between tissue compartments. As with numerous types of cancer of epithelial origin, LM-332 is a key element of the basement membrane barrier (25). As an ECM protein unique to epithelial cells, LM-332 plays a key role in cell adhesion and migration $(8,26)$.

LM-332 is encoded by LAMA3, LAMB3 and LAMC2, which are associated with tumor invasiveness in various types of malignant tumor (27). The $\alpha 3$ chain of LM-332 is essential for the interaction with $\alpha 3 \beta 1$ integrin, which promotes cell adhesion, migration and invasion (28). It was recently demonstrated that upregulation of LAMA3 is associated with poor prognosis in PDAC (17). In our previous study, the increased expression of LAMA3 was significantly associated with worse differentiation, deeper depth of invasion, more advanced stage, and shorter survival time in PDAC (17). In addition, LAMA3 expression was assessed in human PDAC cells and it was revealed that it was significantly higher in PANC1 cells than in H6C7 cells. LAMA3 knockdown had a significant effect on the proliferation of PANC1 cells, as demonstrated by the CCK-8 and colony formation assays. Furthermore, the invasion and migration of PANC1 cells in the Transwell assay were significantly decreased following LAMA3 knockdown.

LAMB3 encodes two transcript variants (29); nevertheless, it seems that only the truncated form of LAMB3 is translated into proteins (30). In prostate carcinoma cell lines, when this variant is expressed (where it is naturally lacking), cell migration and tumorigenicity are promoted $(31,32)$. It was previously indicated that overexpression of LAMB3 is associated with clinicopathological features and decreased survival time of patients with PDAC (20). The results of the present study suggested that LAMB3 expression may play a key role in the progression and prognosis of PDAC. As a potential biomarker of cancer invasion and metastasis, LAMB3 participates in the focal adhesion pathway (33). However, to the best of our knowledge, the function of LAMB3 in pancreatic cancer has not yet been investigated. Similarly, the present study found that LAMB3 knockdown has a significant effect on the proliferation, invasion, migration and EMT of PANC1 cells. Further research is required in order to validate whether loss of LAMB3 expression is associated with methylation at the promoter region and clinicopathological features of poor prognosis in PADC. 
A

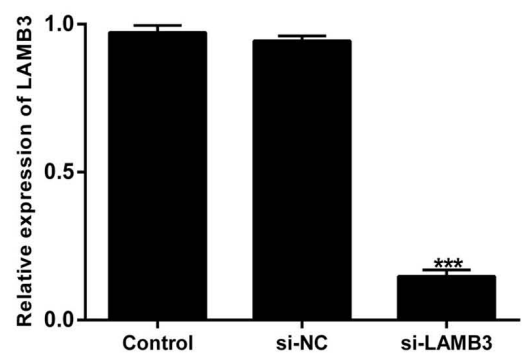

B

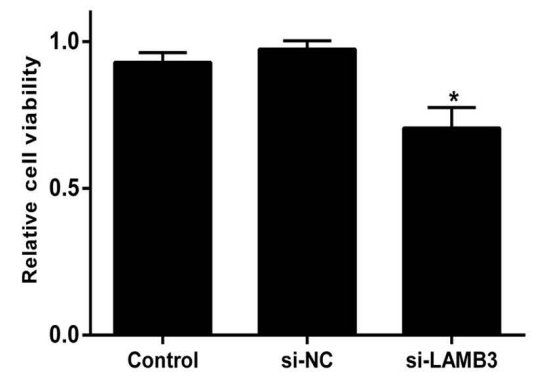

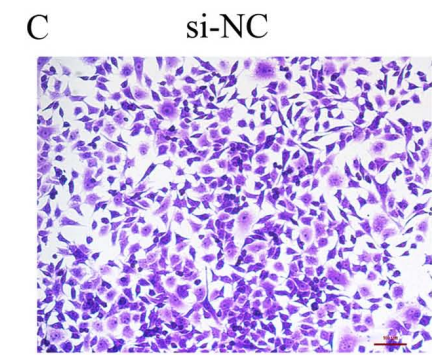

$\mathrm{D}$
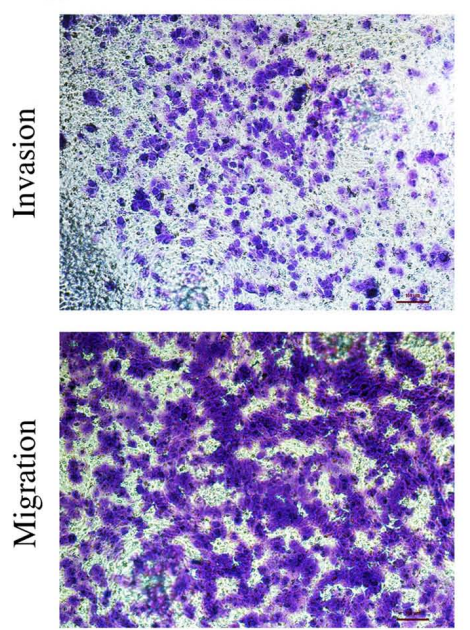

si-LAMB3

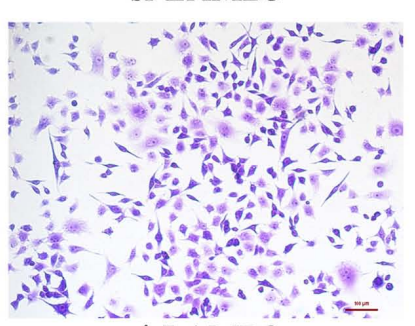

si-LAMB3
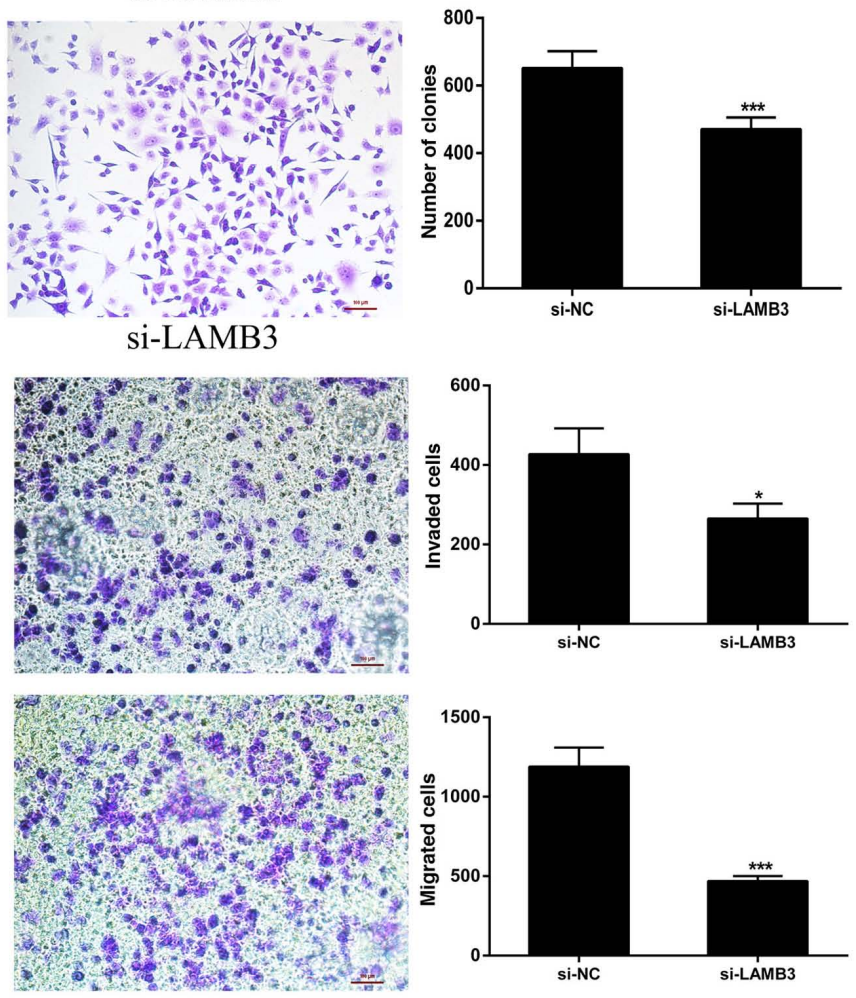

E
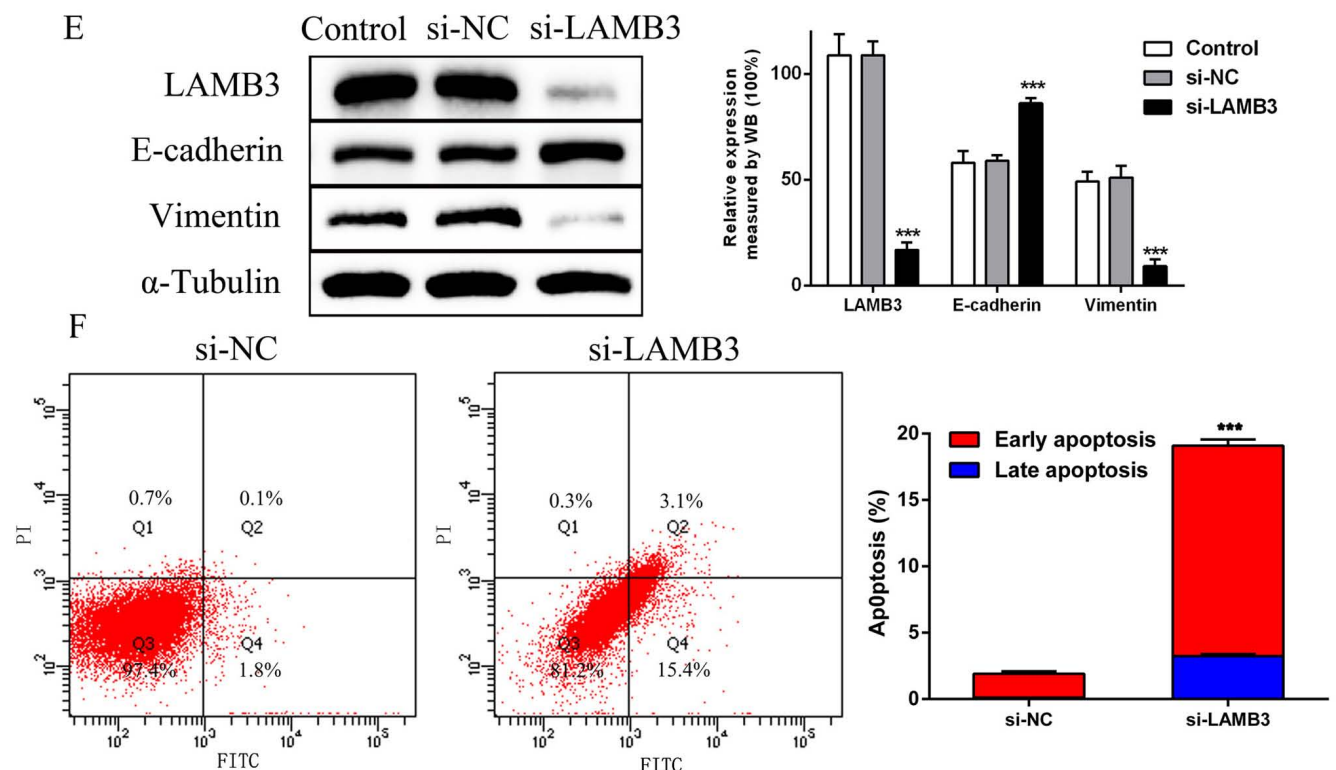

Figure 3. Knockdown of LAMB3 inhibits PDAC cell proliferation, migration, invasion and EMT, and promotes PDAC cell apoptosis. (A) LAMB3 mRNA expression was evaluated via reverse transcription-quantitative PCR in PANC-1 cells transfected with si-LAMB3. (B) Cell proliferation was analyzed by Cell Counting Kit-8 assays in the Control, si-NC and si-LAMB3 groups. (C) Colony formation assays were performed in the si-NC and si-LAMB3 groups. (D) Transwell invasion and migration assays. (E) EMT-associated proteins E-cadherin and vimentin were examined via WB analysis in the Control, si-NC and si-LAMB3 groups. (F) The percentage of apoptotic cells was shown by flow cytometry in the si-NC and si-LAMB3 groups. ${ }^{*} \mathrm{P}<0.05,{ }^{* * * *} \mathrm{P}<0.01 \mathrm{vs}$. si-NC group. PDAC, pancreatic ductal adenocarcinoma; EMT, epithelial-to-mesenchymal transition; siRNA, small interfering RNA; NC, negative control; LAMB3, laminin subunit $\beta 3$; WB, western blotting. 
A

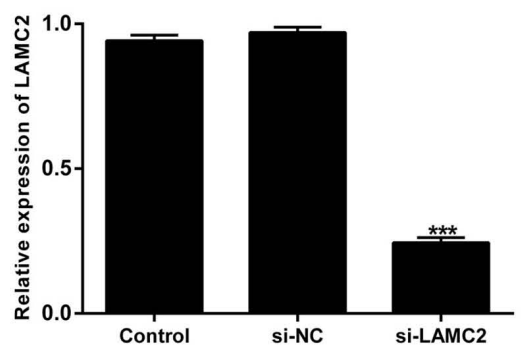

B

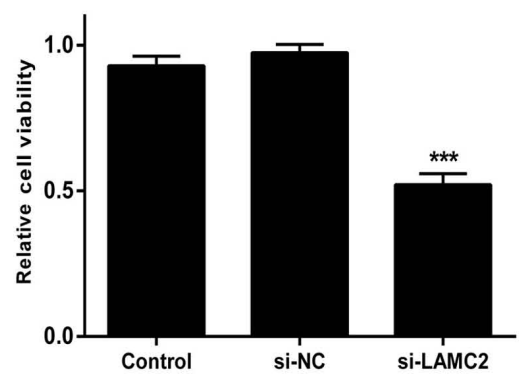

C

si-NC

si-LAMC2

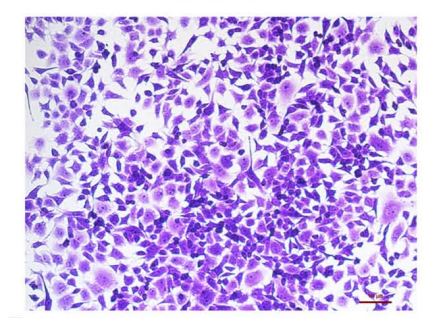

si-NC
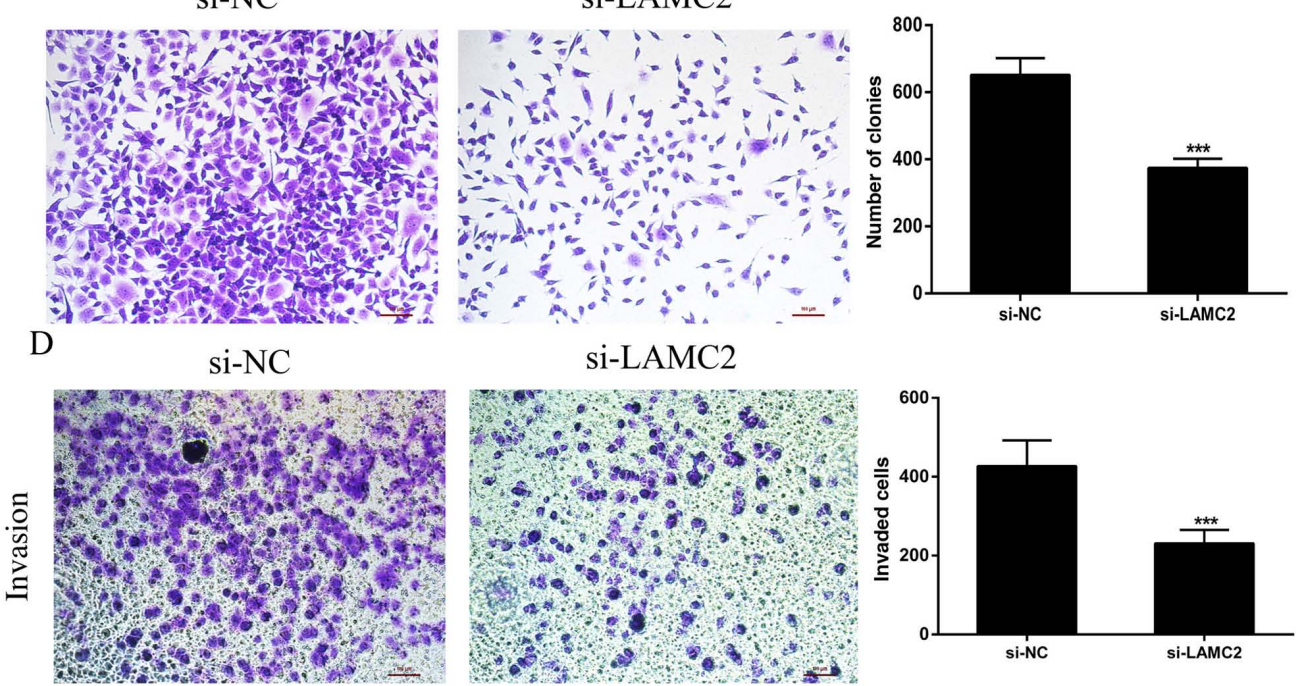

si-LAMC2
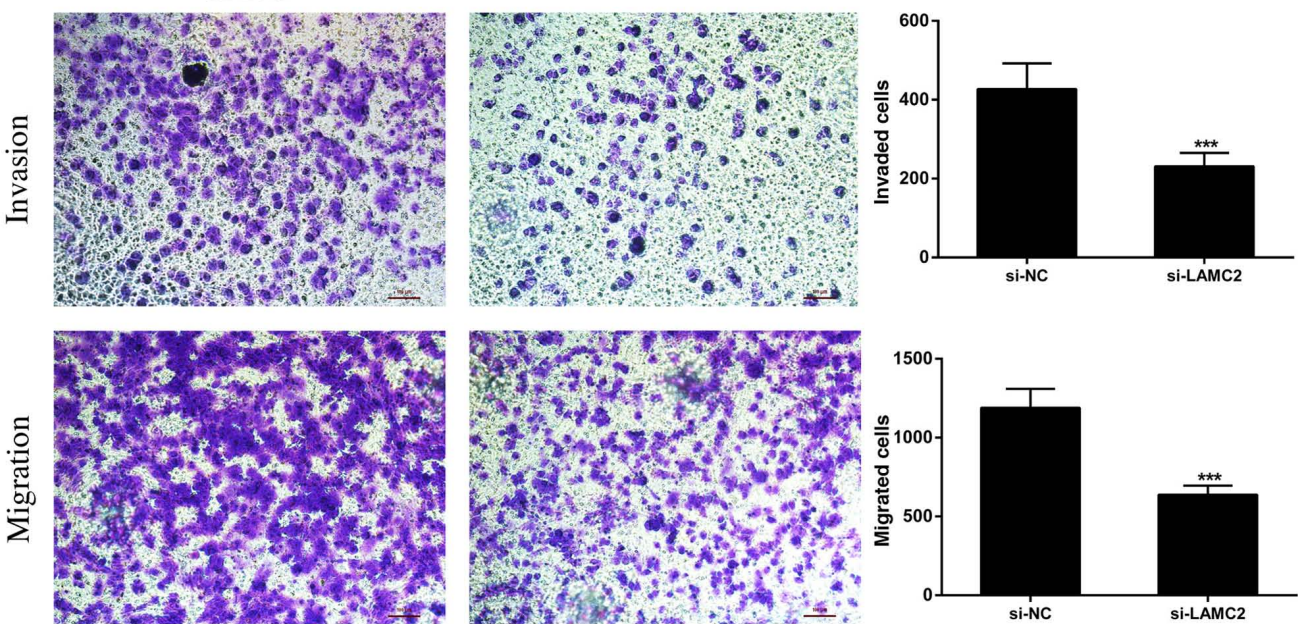

E
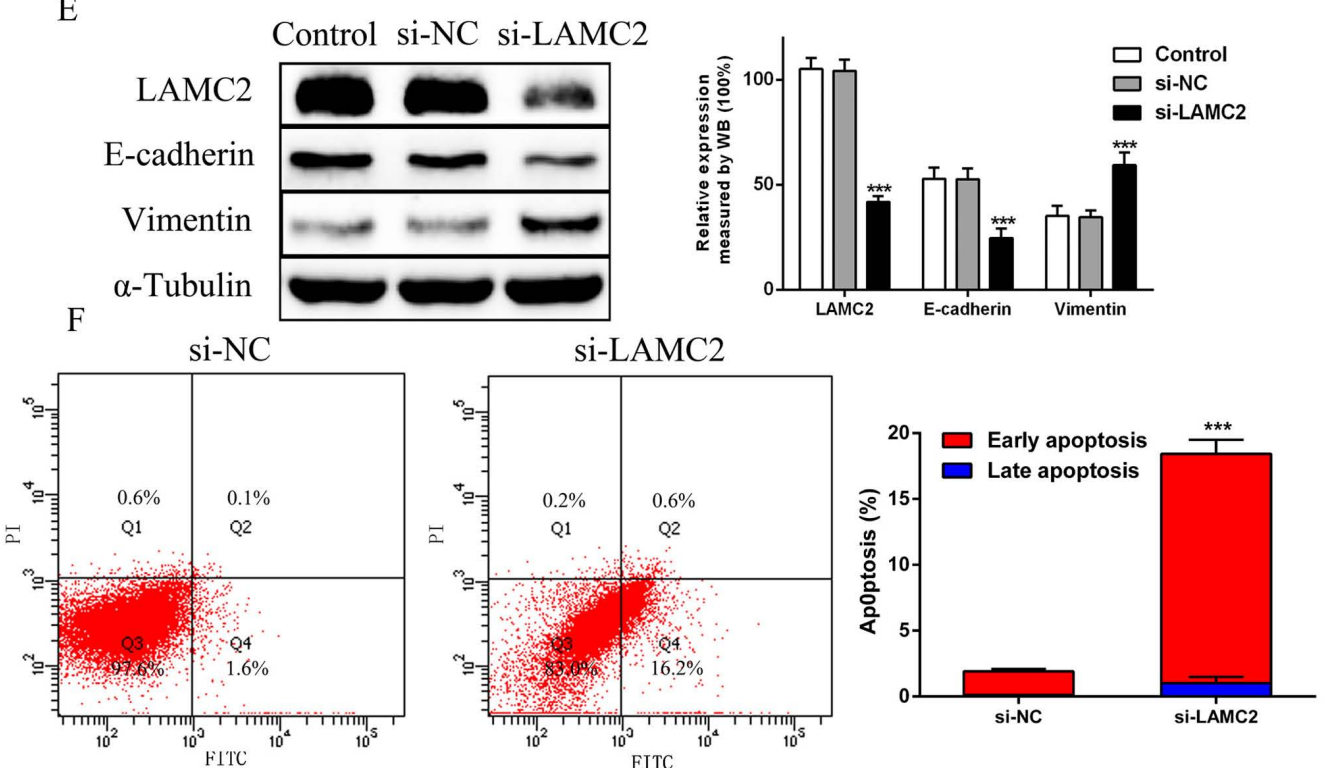

Figure 4. Knockdown of LAMC2 inhibits PDAC cell proliferation, migration and invasion, and promotes PDAC cell apoptosis and EMT. (A) LAMC2 mRNA expression was evaluated via reverse transcription-quantitative PCR in PANC-1 cells transfected with si-LAMC2. (B) Cell proliferation was analyzed by Cell Counting Kit-8 assays in the Control, si-NC and si-LAMC2 groups. (C) Colony formation assays were performed in the si-NC and si-LAMC2 groups. (D) Transwell invasion and migration assays. (E) EMT-associated proteins E-cadherin and vimentin were examined via WB analysis in the Control, si-NC and si-LAMC2 groups. (F) The percentage of apoptotic cells was illustrated by flow cytometry in the si-NC and si-LAMC2 groups. ${ }^{* * * *} \mathrm{P}<0.01$ vs. si-NC group. PDAC, pancreatic ductal adenocarcinoma; EMT, epithelial-to-mesenchymal transition; siRNA, small interfering RNA; NC, negative control; LAMC2, laminin subunit $\gamma 2$; WB, western blotting. 
Research generally suggests that overexpression of LM-332 or one of its subunits (particularly $\gamma 2$ ) is associated with poor prognosis in the majority of cancer types (34). It has been reported that the cytoplasmic expression of LAMC2 demonstrates high invasive potential of tumors and is correlated with distant metastasis, particularly hepatic metastasis, and poor prognosis (27). Similarly, it has recently been demonstrated that overexpression of LAMC2 is associated with poor prognosis in PDAC (17). However, the presence of $\gamma 2$ appears to predict an improved prognosis in breast and colon cancer $(35,36)$. The present study revealed that LAMC2 knockdown had a significant effect on the proliferation, invasion and migration of PANC1 cells. However, si-LAMC2 significantly decreased E-cadherin expression and increased vimentin expression, indicating that si-LAMC2 promoted the progression of EMT. Further research is required in order to validate the transduction pathway and underlying mechanism of LAMC2.

EMT is a process that converts epithelial cells into mesenchymal stem cells, with loss of polarity and adhesion, and gain of migration and invasive properties (37). EMT can enhance the invasive, migratory and metastatic ability of tumor cells, and it can help cells escape apoptosis induced by certain factors in the process of tumor development. However, tumor metastasis involves numerous factors, such as cytokines and the internal microenvironment, and EMT may be one of the most important factors in the overall process (38). Tumor cells collected from bone metastases in patients with prostate, breast and colon cancer expressed EMT markers, and those with ovarian cancer showed EMT in the process of metastasis (39-41).

There may be some limitations of the present study. First, there were no investigations into the effects of LAMA3, LAMB3 and LAMC2 overexpression. Secondly, this study did not explore the underlying mechanism that mediates cell invasion and migration, or EMT regulation by LM-332 in PDAC. Furthermore, there was no investigation into the reason that the three LM-332 subunits $\alpha 3, \beta 3$ and $\gamma 2$, exerted different effects on PDAC cells, nor was there any investigation into the underlying mechanism of this finding, which will form the main focus of future research. In our previous research, enhanced expression of LAMA3, LAMB3 and LAMC2 were confirmed with tissue analysis $(17,20)$. The increased expression levels of the three subunits of LM-332 were significantly associated with worse differentiation, deeper depth of invasion, a more advanced stage and shorter survival time in PDAC. Zhang et al (33) illustrated that LAMB3-mediated invasion and migration may occur through the PI3K/Akt signaling pathway. However, the exact underlying mechanism of LAMA3 and LAMC2 in PACD cells remains unclear, and further studies are required to confirm this hypothesis. We will try to explore functional analysis in a future study. Such as, it will be necessary to confirm co-localization of LAMA3, LAMB3 and LAMC2 in pancreatic cancer cells and to investigate the interaction with specific integrins.

The present study revealed that LAMA3 knockdown was closely associated with cell proliferation in PDAC. In addition, LAMB3 knockdown regulated the EMT-associated proteins E-cadherin and vimentin, indicating that LAMB3 is essential for EMT. Furthermore, LAMC2 was associated with invasion and migration in PDAC. These results may provide potential targets and novel therapeutic strategies for the control of invasion and metastasis in PDAC.

\section{Acknowledgements}

Not applicable.

\section{Funding}

The present work was supported by Science and Technology Bureau of Jinhua City (grant no. 2015-3-005).

\section{Availability of data and materials}

All data generated or analyzed during this study are included in this published article.

\section{Authors' contributions}

JC and $\mathrm{CH}$ contributed to the conceptualization and design of the study. JC drafted and critically revised the work. $\mathrm{CH}$ performed the experiments. All authors read and approved the final manuscript.

\section{Ethics approval and consent to participate}

Not applicable.

\section{Patient consent for publication}

Not applicable.

\section{Competing interests}

The authors declare that they have no competing interests.

\section{References}

1. Fan CS, Chen LL, Hsu TA, Chen CC, Chua KV, Li CP and Huang TS: Endothelial-mesenchymal transition harnesses HSP90 $\alpha$-secreting M2-macrophages to exacerbate pancreatic ductal adenocarcinoma. J Hematol Oncol 12: 138, 2019.

2. Erickson LA: Pancreatic ductal adenocarcinoma. Mayo Clin Proc 92: 1461-1462, 2017.

3. Li JT, Wang YP, Yin M and Lei QY: Metabolism remodeling in pancreatic ductal adenocarcinoma. Cell Stress 3: 361-368, 2019.

4. Ayres Pereira M and Chio IC: Metastasis in pancreatic ductal adenocarcinoma: Current standing and methodologies. Genes (Basel) 11: 11, 2019

5. Li B, Liu B, Zhang X, Liu H and He L: KIF18B promotes the proliferation of pancreatic ductal adenocarcinoma via activating the expression of CDCA8. J Cell Physiol 235: 4227-4238, 2020.

6. Oh KH, Choi J, Woo JS, Baek SK, Jung KY, Koh MJ, Kim YS and Kwon SY: Role of laminin 332 in lymph node metastasis of papillary thyroid carcinoma. Auris Nasus Larynx 44: 729-734, 2017.

7. Kang SG, Ha YR, Ko YH, Kang SH, Joo KJ, Cho HY, Park HS, Kim CH, Kwon SY, Kim JJ, et al: Effect of laminin 332 on motility and invasion in bladder cancer. Kaohsiung J Med Sci 29: 422-429, 2013.

8. Carpenter PM, Sivadas P, Hua SS, Xiao C, Gutierrez AB, Ngo T and Gershon PD: Migration of breast cancer cell lines in response to pulmonary laminin 332. Cancer Med 6: 220-234, 2017.

9. Chiorean R, Danescu S, Virtic O, Mustafa MB, Baican A, Lischka A, Hashimoto T, Kariya Y, Koch M and Sitaru C: Molecular diagnosis of anti-laminin 332 (epiligrin) mucous membrane pemphigoid. Orphanet J Rare Dis 13: 111, 2018. 
10. Rousselle $\mathrm{P}$ and Beck K: Laminin 332 processing impacts cellular behavior. Cell Adhes Migr 7: 122-134, 2013.

11. Carpenter PM, Ziogas A, Markham EM, Cantillep AS, Yan R and Anton-Culver $\mathrm{H}$ : Laminin 332 expression and prognosis in breast cancer. Hum Pathol 82: 289-296, 2018.

12. Lamouille S, Xu J and Derynck R: Molecular mechanisms of epithelial-mesenchymal transition. Nat Rev Mol Cell Biol 15: 178-196, 2014

13. De Craene B and Berx G: Regulatory networks defining EMT during cancer initiation and progression. Nat Rev Cancer 13: 97-110, 2013

14. Wrighton KH: Cell migration: EMT promotes contact inhibition of locomotion. Nat Rev Mol Cell Biol 16: 518, 2015

15. Lee J, Choi JH and Joo CK: TGF- $\beta 1$ regulates cell fate during epithelial-mesenchymal transition by upregulating survivin. Cell Death Dis 4: e714, 2013.

16. Kenda Suster N, Smrkolj S and Virant-Klun I: Putative stem cells and epithelial-mesenchymal transition revealed in sections of ovarian tumor in patients with serous ovarian carcinoma using immunohistochemistry for vimentin and pluripotency-related markers. J Ovarian Res 10: 11, 2017.

17. Chen J, Zhang H, Luo J, Wu X, Li X, Zhao X, Zhou D and Yu S: Overexpression of $\alpha 3, \beta 3$ and $\gamma 2$ chains of laminin-332 is associated with poor prognosis in pancreatic ductal adenocarcinoma. Oncol Lett 16: 199-210, 2018.

18. Murakami S, Shahbazian D, Surana R, Zhang W, Chen H, Graham GT, White SM, Weiner LM and Yi C: Yes-associated protein mediates immune reprogramming in pancreatic ductal adenocarcinoma. Oncogene 36: 1232-1244, 2017.

19. Pandey R, Zhou M, Islam S, Chen B, Barker NK, Langlais $P$ Srivastava A, Luo M, Cooke LS, Weterings E, et al: Carcinoembryonic antigen cell adhesion molecule 6 (CEACAM6) in Pancreatic Ductal Adenocarcinoma (PDA): An integrative analysis of a novel therapeutic target. Sci Rep 9: 18347, 2019.

20. Chen J, Wang W, Wei J, Zhou D, Zhao X, Song W, Sun Q, Huang $\mathrm{P}$ and Zheng $\mathrm{S}$ : Overexpression of $\beta 3$ chains of laminin-332 is associated with clinicopathologic features and decreased survival in patients with pancreatic adenocarcinoma. Appl Immunohistochem Mol Morphol 23: 516-521, 2015

21. Gupta R, Amanam I and Chung V: Current and future therapies for advanced pancreatic cancer. J Surg Oncol 116: 25-34, 2017.

22. Kligys K, Wu Y, Hamill KJ, Lewandowski KT, Hopkinson SB BudingerGR and Jones JC:Laminin-332 and $\alpha 3 \beta 1$ integrin-supported migration of bronchial epithelial cells is modulated by fibronectin. Am J Respir Cell Mol Biol 49: 731-740, 2013.

23. Erdogan B and Webb DJ: Cancer-associated fibroblasts modulate growth factor signaling and extracellular matrix remodeling to regulate tumor metastasis. Biochem Soc Trans 45: 229-236, 2017.

24. Lugano R, Vemuri K, Yu D, Bergqvist M, Smits A, Essand M, Johansson S, Dejana E and Dimberg A: CD93 promotes $\beta 1$ integrin activation and fibronectin fibrillogenesis during tumor angiogenesis. J Clin Invest 128: 3280-3297, 2018.

25. Kamoshida G, Ogawa T, Oyanagi J, Sato H, Komiya E, Higashi S, Miyazaki K and Tsuji T: Modulation of matrix metalloproteinase-9 secretion from tumor-associated macrophage-like cells by proteolytically processed laminin-332 (laminin-5). Clin Exp Metastasis 31: 285-291, 2014.

26. Kariya Y, Sato H,Katou N, Kariya Y and Miyazaki K: Polymerized laminin-332 matrix supports rapid and tight adhesion of keratinocytes, suppressing cell migration. PLoS One 7: e35546, 2012.

27. Guess CM and Quaranta V: Defining the role of laminin-332 in carcinoma. Matrix Biol 28: 445-455, 2009.

28. Zhang J, Wang H, Wang Y, Dong W, Jiang Z and Yang G: Substrate-mediated gene transduction of LAMA3 for promoting biological sealing between titanium surface and gingival epithelium. Colloids Surf B Biointerfaces 161: 314-323, 2018.
29. Fuentes I, Campos M, Repetto G, Morandé P, Yubero MJ, Gonzalez S, Klausegger A, Schnitzhofer P, Pohla-Gubo G, Bauer J, et al: Molecular epidemiology of junctional epidermolysis bullosa: Discovery of novel and frequent LAMB3 mutations in Chilean patients with diagnostic significance. Br J Dermatol 176: 1090-1092, 2017.

30. Mayer B, Silló P, Mazán M, Pintér D, Medvecz M, Has C, Castiglia D, Petit F, Charlesworth A, Hatvani Z, et al: A unique LAMB3 splice-site mutation with founder effect from the Balkans causes lethal epidermolysis bullosa in several European countries. Br J Dermatol 175: 721-727, 2016.

31. Calaluce R, Bearss DJ, Barrera J, Zhao Y, Han H, Beck SK, McDaniel K and Nagle RB: Laminin-5 beta3A expression in $\mathrm{LNCaP}$ human prostate carcinoma cells increases cell migration and tumorigenicity. Neoplasia 6: 468-479, 2004.

32. Jung SN, Lim HS, Liu L, Chang JW, Lim YC, Rha KS and Koo BS: LAMB3 mediates metastatic tumor behavior in papillary thyroid cancer by regulating c-MET/Akt signals. Sci Rep 8: 2718, 2018.

33. Zhang H, Pan YZ, Cheung M, Cao M, Yu C, Chen L, Zhan L, He ZW and Sun CY: LAMB3 mediates apoptotic, proliferative, invasive, and metastatic behaviors in pancreatic cancer by regulating the PI3K/Akt signaling pathway. Cell Death Dis 10: 230 , 2019.

34. Kobayashi T, Masaki T, Nozaki E, Sugiyama M, Nagashima F, Furuse J, Onishi H, Watanabe T and Ohkura Y: Microarray analysis of gene expression at the tumor front of colon cancer. Anticancer Res 35: 6577-6581, 2015.

35. Moon YW, Rao G, Kim JJ, Shim HS, Park KS, An SS, Kim B, Steeg PS, Sarfaraz S, Changwoo Lee L, et al: LAMC2 enhances the metastatic potential of lung adenocarcinoma. Cell Death Differ 22: 1341-1352, 2015.

36. Sato H, Higashi S and Miyazaki K: Amino-terminal fragments of laminin $\gamma 2$ chain stimulate migration of metastatic breast cancer cells by interacting with CD44. Clin Exp Metastasis 32: 405-415, 2015 .

37. Crha K, Ventruba P, Žáková J, Ješeta M, Pilka R, Vodička J and Serpa P: The role of mesenchymal-epithelial transition in endometrial function and receptivity. Ceska Gynekol 84: 371-375, 2019.

38. Kar R, Jha NK, Jha SK, Sharma A, Dholpuria S, Asthana N, Chaurasiya K, Singh VK, Burgee S and Nand P: A 'NOTCH' deeper into the epithelial-to-mesenchymal transition (EMT) program in breast cancer. Genes (Basel) 10: 10, 2019.

39. Peng YS, Syu JP, Wang SD, Pan PC and Kung HN: BSA-bounded p-cresyl sulfate potentiates the malignancy of bladder carcinoma by triggering cell migration and EMT through the ROS/Src/FAK signaling pathway. Cell Biol Toxicol 36: 287-300, 2020.

40. Hanrahan K, O'Neill A, Prencipe M, Bugler J, Murphy L, Fabre A, Puhr M, Culig Z, Murphy K and Watson RW: The role of epithelial-mesenchymal transition drivers ZEB1 and ZEB2 in mediating docetaxel-resistant prostate cancer. Mol Oncol 11: 251-265, 2017.

41. Li L, Liu J, Xue H, Li C, Liu Q, Zhou Y, Wang T, Wang H, Qian H and Wen T: A TGF-beta-MTA1-SOX4-EZH2 signaling axis drives epithelial-mesenchymal transition in tumor metastasis. Oncogene 39: 2125-2139, 2020.

This work is licensed under a Creative Commons Attribution-NonCommercial-NoDerivatives 4.0 International (CC BY-NC-ND 4.0) License. 